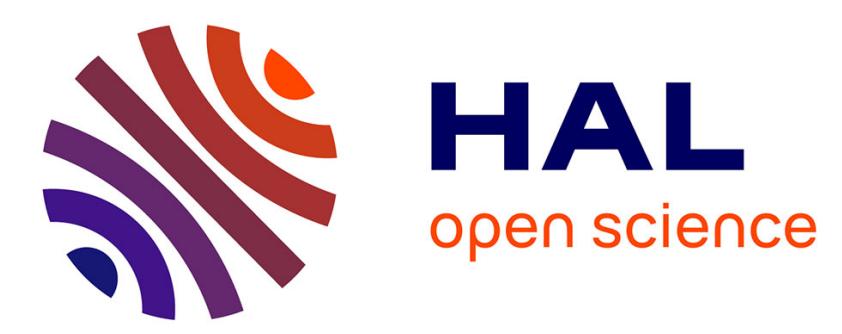

\title{
Magnetic sensor noise impact on the evaluation of magnetorelaxometry experiments
}

Matthieu Denoual, Kamel Fodil, Christophe Dolabdjian, F. Kauffmann

\section{To cite this version:}

Matthieu Denoual, Kamel Fodil, Christophe Dolabdjian, F. Kauffmann. Magnetic sensor noise impact on the evaluation of magnetorelaxometry experiments. International Conference on Noise and Fluctuations (ICNF), 2013, Montpellier, France. hal-00981597

\section{HAL Id: hal-00981597 \\ https://hal.science/hal-00981597}

Submitted on 22 Apr 2014

HAL is a multi-disciplinary open access archive for the deposit and dissemination of scientific research documents, whether they are published or not. The documents may come from teaching and research institutions in France or abroad, or from public or private research centers.
L'archive ouverte pluridisciplinaire HAL, est destinée au dépôt et à la diffusion de documents scientifiques de niveau recherche, publiés ou non, émanant des établissements d'enseignement et de recherche français ou étrangers, des laboratoires publics ou privés. 


\section{Magnetic sensor noise impact on the evaluation of magnetorelaxometry experiments}

\author{
M. Denoual ${ }^{1,3,4}$, K. Fodil $^{1,2,4}$, C. Dolabdjian ${ }^{1,2,4}$ \\ ${ }^{1}$ Normandie Univ, France ${ }^{2}$ UCBN, ${ }^{3}$ ENSICAEN, \\ GREYC, F-14032 Caen, France ; ${ }^{4}$ CNRS, UMR 6072, F- \\ 14032 Caen, France
}

\author{
F. Kauffmann $n^{1,2,3}$ \\ ${ }^{1}$ Normandie Univ, France ; ${ }^{2}$ UCBN, LMNO, F-14032 \\ Caen, France ; ${ }^{4}$ CNRS, UMR 6119, F-14032 Caen, France
}

\begin{abstract}
A complete statistical model taking into account the nature of the magnetic sensor noise is proposed for parameter extraction in magnetorelaxometry experiments on magnetic nanoparticles (MNPs). Apart from the noise, the important factors are the type of the magnetic sensor used as well as the regression model depending on the relaxation mechanism. Whatever the relaxation mechanism is, the models are nonlinear. Consequently, the relation between the signal to noise ratio of the measurement and the uncertainty of the extracted parameters is not straightforward. From the model, we derive a figure of merit that enables an a priori performance extrapolation from the characteristics of the magnetometer.
\end{abstract}

Keywords-magnetorelaxometry, figure of merit

\section{INTRODUCTION}

Magnetic nanoparticles are in use for new applications in the biomedical field. They enable promising developments including manipulation, treatment and detection [1]. They can be used as labels in detection techniques such as ACsusceptibility and magnetorelaxometry experiments [2].

The objective of this work is to evaluate the impact of the nature of the magnetic sensor noise, used during magnetorelaxometry experiments, on the extraction of fitting parameters. The confidence intervals of the parameters extracted from magnetorelaxometry measurements will be used as a figure of merit to allow an a priori comparison of the performance of magnetometers taking into account their intrinsic noise as well as their dimensional characteristics. Two particular magnetometers, the fluxgate and the iGMR (improved Giant MagnetoResistance [3]) magnetometers, were chosen to illustrate the comparison scheme. Those two magnetometers were preferred because of their different nature of noise (white noise and 1/f noise behavior for fluxgate and iGMR, respectively) and their working room temperature. While fluxgates or SQUIDs have been successfully used for several years for magnetorelaxometry experiments [2], iGMR have been more recently introduced in such measurements. In previous work, iGMR were used for magnetorelaxometry experiments for freeze-dried nanoparticles samples [4]. The potential benefits of iGMR magnetometer used for magnetorelaxometry experiments were discussed.

The paper is organized as follows. Firstly, the potential of magnetometers for the magnetorelaxometry experiments is discussed according to their noise characteristics and respective dimensions and bandwidth. A general statistical model for the measured signal taking into account the relaxation mechanism model and noise is presented. From this model, confidence intervals of extracted parameters, used as a figure of merit, are derived in the general case from a multiplicative factor and from the standard deviation of the particular case where the magnetometer exhibiting a white noise. Abacuses are given for the estimation of the confidence interval in the general case.

\section{POTENTIAL OF MAGNETOMETERS FOR MAGNETORELAXOMETRY EXPERIMENTS}

The magnetorelaxometry experiment implies the measurement of the relaxation of magnetic nanoparticles in a sample using a magnetometer in the vicinity of the sample after switching off a magnetizing field. The parameters of interest are extracted from the recorded relaxation curves through fits to non-linear models of the relaxation mechanisms. Therefore, it is obvious that the accuracy of the extracted parameters will depend on the signal-to-noise ratio of the measurement, on the fitting procedure and on the model used. This accuracy can be quantified by the evaluation of a confidence interval associated to the parameters of the given model and signal-to-noise ratio. Meanwhile, the confidence intervals are not straightforward. This paper aims at defining this relation.

Considering the magnetorelaxometry experiments, whatever the relaxation mechanism is, either Néel or Brownian, the measured signal can be expressed by

$$
B_{\text {meas }}(t)=\kappa_{m} \cdot\left[B_{0}+B_{\text {ampl }} \cdot f(t, \tau)\right]+n(t)
$$

where $n(t)$ is the equivalent magnetic noise of the magnetometer, $f(t, \tau)$ the function expressing the relaxation mechanism, $\kappa_{m}$ a coefficient reflecting the gain factor due to the distance between the magnetometer and the sample, and $B_{0}$, $B_{a m p l}$ and $\tau$ are the main parameter to be extracted from the measurement for the characterization of the studied sample. The parameters $\kappa_{m}$ and $n(t)$ depend on the magnetometer used. The proximity effect between the magnetometers and the sample, which depends on the magnetometer size, affects the amplitude of the measured signal, so $\kappa_{m}$, directly. The closer to the sample the magnetometer is, the higher the measured signal is. This is true as far as the magnetometer does not disturb the measurement (e.g., via the feedback circuit or by its own magnetic material). The second term, the noise signal, depends only on the magnetometer and negatively affects the parameter extraction. Depending on the magnetometer, the noise exhibits 
a $1 / f$ contribution (flicker noise) or not. From a general point of view, the higher the noise signal is, the more difficult the parameter extraction is. Thus, the poorer the confidence interval of the extracted parameter will be. But the nature of noise (white noise with or without $1 /$ f noise contribution) shall be taken into account to elaborate the comparison of the figure of merit. Hence, the key point is to determine the relative positive and negative impact of those magnetometer dependent quantities so as to evaluate the potential of magnetometers for magnetorelaxometry experiments.

\section{STATISTICAL MODEL}

Here, we introduce a figure of merit that takes into account the noise contributions of the used magnetometer, especially the $1 / f$ noise contribution. Notice that the hypothesis do not take into account any offset drift during the observation duration. This figure of merit is a multiplicative coefficient that can be used to derive the confidence interval in the general case from the confidence interval in the particular case (the magnetometer has a white noise behavior). Confidence intervals in the latter case have been previously proposed in [4].

\section{A. Noise model}

In order to estimate and to obtain statistical properties of estimated parameters $B_{a m p l}$ and $B_{0}$, we propose to model noise of magnetometer, $n(t)$, with a parametric family of stationary Gaussian noise $n_{\alpha, d, \sigma}$ up to second order depending on three parameters $\alpha \in[0,1], d \in[0,1 / 2]$ and $\sigma \in[0,+\infty]$, whose power spectral density with the normalized frequency $f \in[-1 / 2,1 / 2]$, is defined by

$$
S_{n \alpha, d, \sigma}(f)=\sigma^{2}\left(\left(1-\alpha^{2}\right)+\alpha^{2} \frac{1 / K_{d}}{\left|1-e^{-i 2 \pi f}\right|^{2 d}}\right)
$$

with $K_{d}=\int_{-1 / 2}^{1 / 2} \frac{1}{\left|1-e^{-i 2 \pi f}\right|^{2 d}} d f$.

In the time domain, we can find two independent white Gaussian noise contributions of unit variances $\varepsilon_{1}, \varepsilon_{2}$ such that

$$
n_{\alpha, d, \sigma}=\sigma\left(\sqrt{1-\alpha^{2}} \varepsilon_{1}+\frac{\alpha}{\sqrt{K_{d}}}\left(1-z^{-1}\right)^{-d} \varepsilon_{2}\right)
$$

where $z^{-1}$ is a delay operator, and $\left(1-z^{-1}\right)^{-d}$ is a fractional integrated operator, $\left(1-z^{-1}\right)^{-d} \varepsilon_{2}$ is in a AutoRegressive Fractionally Integrated Moving Average process named $\operatorname{ARFIMA}(0, \mathrm{~d}, 0)[5-8]$.

The interpretations of these three parameters are the following: $\sigma^{2}$ is the variance of $n(t)$, as the power spectral density is a decreasing function of $f$ in the interval $[0,1 / 2]$, it means that the level of power density of white noise of $n_{\alpha, d, \sigma}$ can be defined by its value for $f=1 / 2$ denoted $W_{\alpha, d, \sigma}=\sigma^{2}\left(\left(1-\alpha^{2}\right)+\frac{\alpha^{2}}{K_{d} 2^{2 d}}\right)$. Therefore $\left(1-\alpha^{2}\right)$ can be interpreted as an approximation of the percentage of the power of white noise which is included in the total power of the power spectral density. When $\alpha=0$, one finds that $n_{\alpha=0, d, \sigma}=\sigma \varepsilon_{1}$ and $S n_{\alpha=0, d, \sigma}(f)=\sigma^{2}$ which means $n_{\alpha=0, d, \sigma}(t)$ is a white Gaussian noise of variance, $\sigma^{2}$, without any $1 / f$ contribution at $f=0^{+}$. When $\alpha=1, n_{\alpha=1, d, \sigma}$ is a purely autogressive, fractionary integrated and moving average. This noise models the noise contribution of magnetometers exhibiting a $1 / f$ noise behavior.

In fact, if $\alpha>0$ then $n_{\alpha, d, \sigma}$ is considered as a long memory process because

$$
\begin{aligned}
& S_{n \alpha, d, \sigma}(f) \approx \frac{\sigma^{2}}{(2 \pi)^{2 d}} \frac{\alpha^{2} / K_{d}}{f^{2 d}}, f \rightarrow 0^{+} \\
& \log \left(S_{n \alpha, d, \sigma}(f)\right) \approx-2 d \log (f), f \rightarrow 0^{+} .
\end{aligned}
$$

Therefore, the parameter $2 d$ represents the slope of power function in a log-log scale. The frequency corner, $f_{\text {corner }}=\frac{1}{2 \pi}\left(\frac{\sigma^{2} \alpha^{2} / K_{d}}{W_{\alpha, d, \sigma}}\right)^{1 /(2 d)}$, of a noise $n_{\alpha, d, \sigma}$ is the frequency for which the asymptote $\left(f \rightarrow 0^{+}\right)$meets the noise density baseline $S=W_{\alpha, d, \sigma}$, as illustrated in Fig. 1 in the case $W_{\alpha, d, \sigma}=1$.

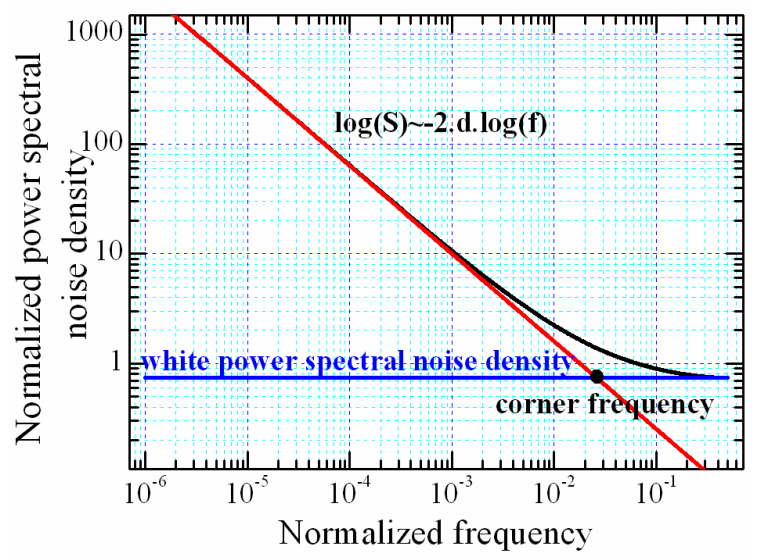

Fig. 1: Normalized power spectral noise density for $n_{\alpha=0.1, d=0.6, \sigma=1}$.

There is a one-to-one correspondence for a fixed $d$ between the parameter $\alpha$ and the corner frequency. It means that the knowledge of the corner frequency allows to derive the parameter $\alpha$. The autocovariance function for $h \in \mathrm{Z}$ of noise $n_{\alpha, d, \sigma}$ is given by

$$
\gamma_{n_{\alpha, d, \sigma}}(h)=\sigma^{2}\left(\left(1-\alpha^{2}\right) \delta_{0}(h)+\alpha^{2} \frac{\Gamma(h+d) \Gamma(h-d)}{\Gamma(h-d+1) \Gamma(d)}\right)(4)
$$

where $\delta_{0}$ is the Dirac function and $\Gamma$ is the gamma function.

\section{B. Regression model}

To estimate the unknown parameters, we propose to use an Expectation-Maximization (EM) type algorithm, which can be described with the following steps:

- searching an admissible solution, and whereas the parameters are not converging,

- maximize the likelihood of the deterministic model knowing the stochastic error model, 
- maximize the likelihood of the stochastic error model knowing the deterministic model.

Let $t_{i}\left(=t_{\text {delay }}+i / F_{e}\right)_{0 \leq i \leq \mathrm{N}}$ be the observation times in second, where $F_{e}$ is the sampling frequency and $t_{\text {delay }}$ is the delay between the beginning of the relaxation and the beginning of the acquisition, then the measured signal can be modeled by

$$
B_{\text {meas }}\left(t_{i}\right)=\kappa_{m} B_{0}+\kappa_{m} B_{\text {ampl }} f\left(t_{i}, \tau\right)+n_{\alpha, d, \sigma}\left(t_{i}\right), 0 \leq i \leq N(5)
$$

Let $z(i, t)=\left(\begin{array}{c}1 \\ f\left(t_{i}, \tau\right)\end{array}\right)$, then the model can be written for $0 \leq \mathrm{i} \leq \mathrm{N}$ as

$$
B_{\text {meas }}\left(t_{i}\right)=z(i, \tau)^{\prime} \beta+n_{\alpha, d, \sigma}\left(t_{i}\right)
$$

where $\beta=\left(\begin{array}{l}\beta_{0} \\ \beta_{1}\end{array}\right)$ is the column vector of the two unknown parameters with $\beta_{0}$ representing $\kappa_{m} B_{0}$ and $\beta_{l}$ representing $\kappa_{m} B_{\text {ampl }} . \tau$ is a vector of parameters of the relaxation model which is supposed to be known. As the model of noise covariance matrix is parametric, depending on the parameters, $\alpha, d, \sigma$, which are assumed to be known, the estimation of the unknown parameters $\beta_{0}$ and $\beta_{l}$ can be derived using the weighted least square method (best linear unbiased estimator).

Classically, let $N$ be the number of observed samples and $\Sigma_{\mathrm{N}}=\operatorname{cov}\left(\left(\mathrm{n}_{\alpha, \mathrm{d}, \sigma}(0), \ldots, \mathrm{n}_{\alpha, \mathrm{d}, \sigma}(\mathrm{N})\right)^{\prime}\right)$ the covariance matrix of the first $N+1$ samples of the noise model $\left(n_{\alpha, d, \sigma}(0), \ldots, n_{\alpha, d, \sigma}(N)\right)$ is given by

$$
\Sigma_{N}=\left(\begin{array}{ccc}
\gamma_{n}(0) & \cdots & \gamma_{n}(N) \\
\vdots & \vdots & \vdots \\
\gamma_{n}(N) & \cdots & \gamma_{n}(0)
\end{array}\right) .
$$

Let $Y_{N}$ be the first observed $N+1$ samples of a relaxation signal. The design matrix $Z_{N}$ of the regression model can be written as

$$
Z_{N}=\left(\begin{array}{cc}
1 & f\left(t_{0}, \alpha\right) \\
\vdots & \vdots \\
1 & f\left(t_{N}, \alpha\right)
\end{array}\right) .
$$

Then, if the rank of $Z_{N}=2$, the Best Linear Unbiased Estimator (BLUE) is uniquely defined by

$$
\hat{\beta}_{\text {BLUE }}=\left(Z_{N}^{\prime} \Sigma_{N}^{-1} Z_{N}\right)^{-1} Z_{N}^{\prime} \Sigma_{N}^{-1} Y_{N} .
$$

With the hypothesis that the physical parameters $\tau$ and parameters $\alpha, d, \sigma$ of the noise model are known, the estimated parameters $\hat{\beta}_{\mathrm{BLUE}}$ is a Gaussian vector with mean $\beta$ (the true one) and covariance matrix

$$
\operatorname{cov}\left(\hat{\beta}_{\text {BLUE }}\right)=\left(Z_{N}^{\prime} \Sigma_{N}^{-1} Z_{N}\right)^{-1}=\left(\begin{array}{cc}
\operatorname{var}\left(\hat{\beta}_{0}\right) & \operatorname{cov}\left(\hat{\beta}_{0}, \hat{\beta}_{1}\right) \\
\operatorname{cov}\left(\hat{\beta}_{1}, \hat{\beta}_{0}\right) & \operatorname{var}\left(\hat{\beta}_{1}\right)
\end{array}\right) .(10)
$$

So, we can define a figure of merit of all magnetometers by the standard deviation $\sigma_{\kappa_{m} B_{a m p l}}(\alpha, d, \sigma)$ of the second term of $\hat{\beta}_{\mathrm{BLUE}}$ which estimates the precision which can be obtained to estimate the concentration $\kappa_{m} B_{\text {ampl }}$. This figure of merit is given by

$$
\sigma_{\kappa_{m} B_{a m p l}}=\sqrt{\operatorname{cov}\left(\hat{\beta}_{B L U E}\right)_{2,2}} .
$$

The latter is independent of the unknown coefficients $\beta$, it only depends on $\sigma_{\kappa_{m} B_{\text {ampl }}}\left(\alpha, d, \sigma, f, \tau, F_{e}, N, t_{\text {delay }}\right)$, i.e.: the characteristics of magnetometer noise $\alpha, d, \sigma$, the geometry of the whole sample and the magnetometer $\kappa_{m}$, the relation function $f$ and its parameter $\tau$ and acquisition parameters $F_{e}, N, t_{\text {delay. }}$.

\section{Figure of merit}

As a figure of merit of a magnetometer, we propose the following criteria

$$
\begin{aligned}
& \operatorname{Merit}\left(\alpha, d, \sigma, f, \tau, F_{e}, N, t_{\text {delay }}\right)= \\
& \quad \sigma_{B_{\text {ampl }}}\left(\alpha, d, \sigma(\alpha, d), f, \tau, F_{e}, N, t_{\text {delay }}\right)
\end{aligned}
$$

where the standard deviation $\sigma(\alpha, d)$ is chosen such that the power density at the highest frequency is one.

This figure of merit of magnetometer can be interpreted as a merit ratio between this magnetometer and the same magnetometer with no $1 / f$ contribution $(\alpha=0)$. It expresses for the impact of $1 / f$ noise contribution on the precision of the estimation of the unknown parameters is $x$ times worse compared to the precision of estimation in the case of a magnetometer having the same white noise level. A magnetometer exhibiting only white noise has a figure of merit of 1 , in particular, its figure of merit does not depend on the relaxation function, whereas the figure of merit for magnetometer exhibiting a $1 / f$ noise contribution depends on the relaxation function

$\operatorname{Merit}\left(\alpha, d, \sigma, f, \tau, F_{e}, N, t_{\text {delay }}\right)=\frac{\sigma_{\kappa_{m} B_{\text {anpl }}}\left(\alpha, d, \sigma(\alpha, d), f, \tau, F_{e}, N, t_{\text {delay }}\right)}{\sigma_{\kappa_{m} B_{\text {anpl }}}\left(\alpha=0, d, \sigma=1, f, \tau, F_{e}, N, t_{\text {delay }}\right)}$

as illustrated in Fig. 3.

\section{Experimental performance comparison}

Fluxgate and iGMR magnetometers are used as examples. The relaxation model for liquid samples (mobile magnetic nanoparticles) is taken as

$$
f(t, \tau)=\exp \left(-\left(\frac{t-\tau_{1}}{\tau_{2}}\right)^{\tau_{3}}\right)
$$

with $\tau_{1}=0 \mathrm{~s}, \tau_{2}=1.28 \mu \mathrm{s}, \tau_{3}=0.23$. We simulate $N=2048$ samples with a sampling frequency $F_{e} \approx 100$ or $200 \mathrm{kHz}$ and a null delay $t_{\text {delay }}=0$. These values correspond to experimental data. 
For the relaxation of freeze-dried samples (immobilized magnetic nanoparticles) the following model is given by

$$
f(t, \tau)=\ln \left(1+\frac{\tau_{2}}{t-\tau_{1}}\right) .
$$

with $\tau_{1}=0 \mathrm{~s}$ and $\tau_{2}=2 \mathrm{~s}$. We simulate $N=4096$ samples with a sampling frequency $F_{e}=5 \mathrm{kHz}$ and a delay $t_{\text {delay }}=1 / F_{e}$. These values correspond to the experimentally realized data presented in previous work [4].

As noise for the used fluxgate magnetometer is nearly white in the considered range of frequency, we can take the model for noise $n_{\alpha, d, \sigma}$ (white noise of variance $\sigma^{2}$ ). For the iGMR noise, we can take the family noise $n_{\alpha, d, \sigma}$ with a fixed $d, \alpha \in[0,1]$, which has a variance of $\sigma^{2}$.

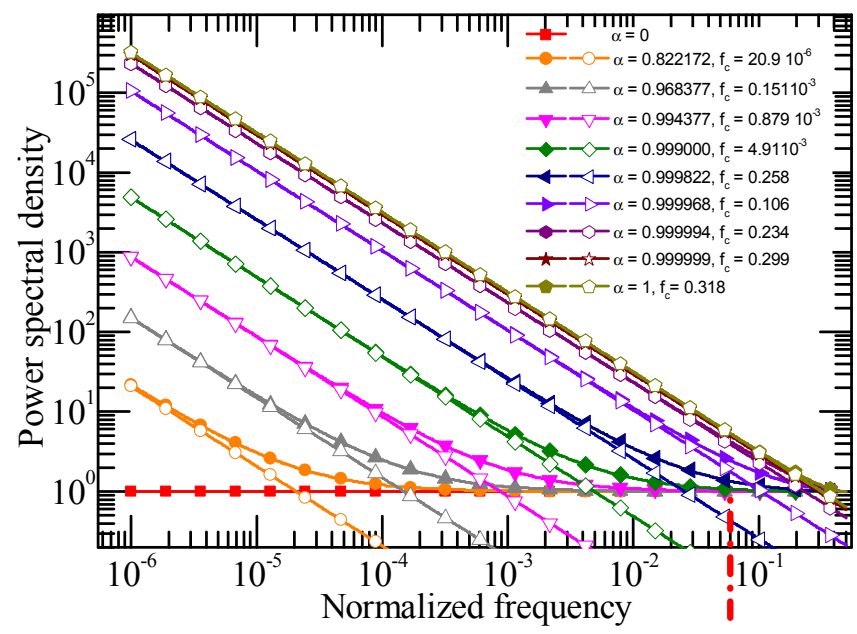

Fig. 2: Normalized power spectral noise density versus normalized frequency for $1 / f$ noise modeling.

As the fluxgate noise is white, the figure of merit of this magnetometer is one. For iGMR magnetometers, we can study the figure of merit in the case of liquid and freeze-dried samples. The parameter $\alpha$ is obtained from Fig. 2 after normalization of the corner frequency experimentally observed. For example, an iGMR used for experiments with a $200 \mathrm{kHz}$ sampling frequency and a noise corner frequency of $10 \mathrm{kHz}$, would have a normalized corner frequency equals to 0.05 . Knowing this value the closest curve in the abacus Fig. 2 gives a parameter $\alpha$ of 0.999822 . The figure of merit is then obtained from Fig. 3. Fig. 3.a. corresponds to the case of liquid samples relaxing via the Brownian mechanism and studied in this paper while Fig. 3.b corresponds to the case of freeze-dried samples. For example, considering the case of liquid sample, and an iGMR magnetometer, the normalized corner frequency derived from experiments implies a 1.33 multiplication factor to derive the standard deviation compared to a magnetometer with the same white noise level and no $1 / f$ contribution. As mentioned previously, in the case of magnetometers exhibiting a $1 / f$ noise contribution, the figure of merit also depends on the relaxation mechanism. Especially, the simulation study indicates that the $1 / f$ noise contribution in magnetometer noise has less impact on experiments with liquid samples than with freeze-dried samples.

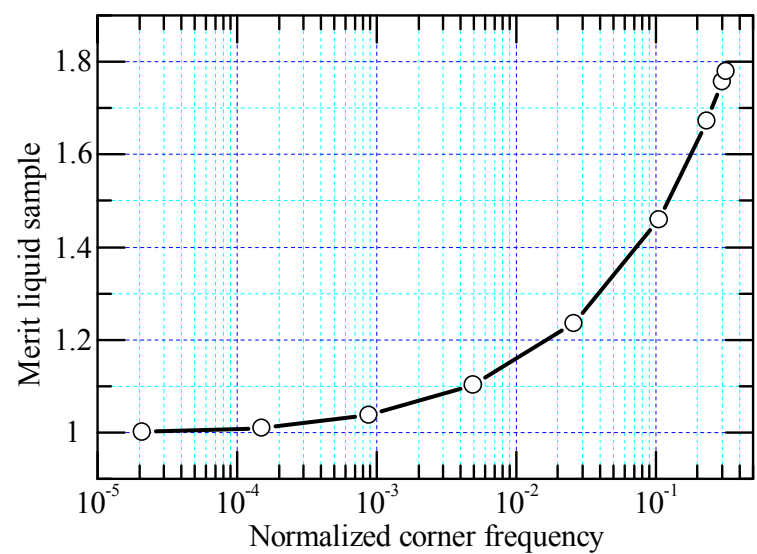

(a)



(b)

Fig. 3: Figure of merit depending on relaxation mechanism for magnetometer exhibiting 1/f noise contribution: case of liquid sample a), case of freeze-dried samples b).

\section{CONCLUSION}

A model that takes into account the nature of the noise of magnetic sensor is proposed. It enables the evaluation of the confidence interval of the parameter extracted during magnetorelaxometry experiments.

\section{References}

[1] E. Parton, G. Borghs, R. De Palma, "Biomedical applications using magnetic nanoparticles", Solid State Technology 50 8, 2007.

[2] F. Ludwig, E. Heim, S. Mauselein, D. Eberbeck, M. Schilling, "Magnetorelaxometry of magnetic nanoparticles with fluxgate magnetometers for the analysis of biological targets”, J. Magn. Magn. Mat. 293, pp. 690-695, 2005

[3] L. Perez, C. Dolabdjian, G. Waché, L. Butin, “Advance in magnetoresistance magnetometer performances applied in eddy current sensor arrays", 16 $6^{\text {th }}$ WCNDT'04 Conference, Montréal, 2004.

[4] M. Denoual, S. Saez, F. Kauffman, C. Dolabdjian, "Magnetorelaxometry using Improved Giant MagnetoResistance Magnetometer", Sensors and Actuators A: Physical, 159, pp. 184-188, 2010.

[5] P.J. Brockwell, R.A. Davis, Times series: theory and methods, Springer Verlag, 2.1, 1990.

[6] M. Taniguchi, Y. Kakizawa, Asymptotic theory of statistical inference for time series, Springer Verlag, 2.5, 2000.

[7] J. Beran, Statistics for Long-Memory Processes, Monographs on Statistics and Applied Probability 61, Chapman \& Hall, NY, 2.1, 2.5, 1994.

[8] J.R.M. Hosking, Fractional differencing, Biometrika, 68, 2.1, 1981. 